\title{
Servicios y formación de los técnicos superiores en audiología protésica en España:una comparación con el modelo de audiólogo general
}

\author{
Carlos R. Benítez-Barrera (1)*1† \\ 'Vanderbilt University, Hearing and Speech Sciences Department, Nashville, TN (EE.UU) \\ Beatriz de Diego-Lázaro (iD ${ }^{2 \dagger}$ \\ 2Midwestern University, Department of Speech and Language Pathology, Glendale, AZ, (EE.UU)
}

\section{¿ OPEN ACCESS \\ PEER REVIEWED}

ARTÍCULO ORIGINAL

Recibido: 07.10.2021

Revisado: 02.11.2021

Aceptado: 22.11.2021

Publicado: 30.12 .2021

\section{Edición}

Miriam I. Marrufo-Pérez

Universidad de Salamanca, España

Revisión

Francesc Roca Ribas

Hospital Universitari Germans Trias i Pujol, España

Daphne Marfull

Universidad del Valparaiso, Chile

Jose Luis Blanco

Demant Ibérica SA, España

\section{Citar como}

Benitez-Barrera, C. R., \& De Diego-Lázaro, B. Servicios y Formación de los Técnicos Superiores en Audiología Protésica: Una Comparación con el Modelo de Audiólogo General. Auditio. 5(3), e78. https://doi.org/10.51445/sja.auditio.vol5.2021.0078

\section{*Correspondencia}

Carlos R. Benítez-Barrera está ahora en Callier Center for Communication Disorders en la University of Texas at Dallas, 1966 Inwood Road, Dallas, TX, 75235 email: carlos.benitez-barrera@utdallas.edu

\section{†Contribución por igual}

(c) (i) CC-ND-BY 4.0

(C) 2021 Los autores / The authors

\section{Resumen}

La titulación de Técnico Superior en Audiología Protésica (TSAP) nació en España en el año 2001. Desde entonces, han surgido títulos propios de posgrado en audiología. Sin embargo, no existe aún en España el grado universitario de audiología general. El objetivo de este estudio fue examinar los lugares de trabajo, los servicios ofrecidos y la percepción de la formación de los TSAP con y sin formación universitaria y si éstos ejercían labores propias del audiólogo general propuesto por la Federación Europea de Sociedades Audiológicas (EFAS).

Un cuestionario en línea fue completado por 255 TSAP. Se utilizaron análisis de varianza para comparar las respuestas entre TSAP sin formación complementaria, TSAP con grado de una disciplina afín a la audiología, TSAP con posgrado en audiología, y TSAP con titulaciones complementarias.

Independientemente de su nivel educativo, la mayoría de los TSAP indicaron desempeñar su labor profesional en centros auditivos adaptando audífonos a pacientes adultos, para lo cual percibieron estar suficientemente formados. Los TSAP con mayor nivel educativo indicaron trabajar en centros más especializados, así como atender a pacientes pediátricos con más frecuencia, y percibieron que su formación en implantes cocleares era mejor que los TSAP.

En general, los TSAP no reciben formación ni ofrecen servicios en áreas que están dentro de las competencias del audiólogo general, tales como la programación de implantes cocleares o el diagnóstico de problemas vestibulares. Los estudios futuros deberán enfocarse en conocer la formación que reciben los profesionales que ofrecen este tipo de servicios audiológicos en España.

\section{Palabras clave}

Técnico Superior en Audiología Protésica, audiología, formación, educación, servicios audiológicos

\section{Implicaciones Clínicas}

En España, la mayoría de los técnicos superiores en audiología protésica trabajan en centros auditivos y ópticas donde adaptan audífonos a pacientes adultos. En este entorno laboral implementan buenas prácticas y se sienten competentes para realizar su práctica clínica. Sin embargo, pese a que algunos de ellos reciben formación universitaria especializada en audiología, no desempeñan labores propias del audiólogo general según el modelo de la Federación Europea de Sociedades Audiológicas (EFAS). Esto puede tener consecuencias a nivel clínico, ya que no se conoce en la actualidad qué profesionales llevan a cabo las tareas del audiólogo general, ni la calidad de su formación, o la calidad del servicio ofrecido. Se requiere un análisis mas detallado de la situación de la audiología en España en cuanto a la provisión de servicios audiológicos clínicos y las competencias profesionales para determinar si es necesario implementar formalmente la figura de audiólogo general en España. 


\section{Introducción}

El aumento de la prevalencia de la pérdida auditiva en la sociedad ha generado la necesidad de incrementar el número de profesionales y de programas de formación en el ámbito de la audiología (Goulios, 2010; Goulios \& Patuzzi, 2008). Debido a esta necesidad y tras diagnosticar una situación precaria en los servicios de salud auditiva, la Federación Europea de Sociedades Audiológicas (EFAS, por sus siglas en inglés) publicó en el año 2001 el modelo de audiólogo general $(A G)$, el cual recomendó implementar a los distintos países europeos (EFAS, 2001). Esta iniciativa tuvo como objetivo crear una nueva figura profesional que tuviera competencias para proveer servicios audiológicos que hasta ese momento eran proporcionados por una amalgama de profesionales de la salud (p. ej., otorrinolaringólogos [ORL], médicos audiólogos ${ }^{1} \mathrm{o}$ audiólogos protésicos). Estas competencias incluían el diagnóstico y tratamiento de la pérdida auditiva, así como la evaluación y el tratamiento de acúfenos o trastornos vestibulares (EFAS, 2001). Debido a la perspectiva generalista del AG, las competencias de los ORL, los médicos audiólogos y los audiólogos protésicos se solapan parcialmente con las del AG. Por ejemplo, mientras que el AG y el ORL tienen competencias para llevar a cabo una otoscopia, solo el ORL está capacitado para tratar afecciones del oído externo y medio. Cabe destacar que las competencias específicas de cada uno de estos profesionales pueden variar según la legalidad vigente en cada país. De cualquier manera, la evaluación y el tratamiento audiológico debe llevarlos a cabo un equipo interdisciplinar. Se recomienda que sea el AG el coordinador del equipo interdisciplinar, delegando aspectos médicos y quirúrgicos del tratamiento en los ORL y/o los médicos audiólogos, y ejerciendo una labor de supervisión sobre el trabajo del audiólogo protésico (American Speech-LanguageHearing Association, 2020; EFAS, 2001). Por ello, el AG puede desempeñar su labor profesional en multitud de entornos laborales, desde centros auditivos especializados en la adaptación de audífonos hasta clínicas privadas de ORL $u$ hospitales centrados en la implantación coclear.

El médico audiólogo es una figura profesional que no existe en España, pero si en otros paĺses europeos y algunos paĺses de Hispanoamérica. El médico audiólogo recibe una formación de base en medicina y después se especializa en audiologĺa, sin ser necesariamente ORL.
En España, las recomendaciones de la EFAS desencadenaron la creación de un título de ciclo superior $^{2}$ de Técnico Superior en Audiología Protésica (TSAP) en el año 2001, el cual no existía hasta la fecha (Real Decreto 1685/2007); sin embargo, se consideró innecesario crear un programa oficial universitario (de grado o de posgrado) en audiología general. Se dispuso que el diagnóstico y el tratamiento de la pérdida auditiva a través de audífonos podrían llevarlo a cabo TSAP, mientras que otros servicios audiológicos no relacionados con la adaptación de audífonos (p. ej., implantes cocleares) continuarían siendo ofrecidos por profesionales de disciplinas afines a la audiología, principalmente el ORL y otros profesionales del ámbito médico (Orden SCO/1262/2007).

Pese a la falta de reconocimiento del AG, han ido surgiendo en España en los últimos años posgrados universitarios cuyo plan de estudios reproduce, al menos parcialmente, el modelo de AG; estos posgrados toman la forma de títulos propios, por lo que, pese a proveer formación especializada y acreditada, no capacitan oficialmente para ejercer la profesión de AG. Debido a ello, la titulación y la figura profesional del AG nunca ha existido formalmente en España. La aparición de estos posgrados ha causado que existan actualmente en España cuatro perfiles profesionales de TSAP: profesionales en posesión únicamente del ciclo superior en audiología, profesionales con ciclo superior y posgrado en audiología, profesionales con ciclo superior en audiología y grado universitario ${ }^{3}$ de una disciplina afín a la audiología $y$, por último, profesionales con ciclo superior en audiología, grado universitario de una disciplina afín y posgrado en audiología. El objetivo de este estudio fue comparar los lugares de trabajo, los servicios ofrecidos y la percepción sobre su formación de los distintos perfiles de los TSAP existentes actualmente en España, y examinar si alguno de estos perfiles profesionales realizaba labores propias del AG. Nuestra hipótesis fue que los TSAP con grado universitario de una disciplina afín y/o un posgrado en audiología mostrarían mejor percepción de su

${ }^{2}$ Los ciclos formativos superiores son estudios profesionales que proporcionan una alternativa a la universidad, imparten formación práctica y permiten la incorporación rápida al mercado laboral.

Desde el año 2007 y con la implantación de los llamados planes de Bolonia, las titulaciones universitarias en España reciben el nombre de "grados", aunque en este artículo incluimos también bajo este término a aquellos profesionales que recibieron su titulación universitaria en planes anteriores a la reforma de Bolonia. 
formación y mayores competencias profesionales que aquellos que solo estuvieran en posesión del ciclo de TSAP. Además, hipotetizamos que los TSAP con grado universitario de una disciplina afín y/o un posgrado en audiología desempeñarían su labor profesional con mayor frecuencia en lugares de trabajo fuera de los centros auditivos u ópticas, y que ofrecerían un abanico de servicios más amplio que los TSAP con solo el ciclo superior en audiología. Por lo tanto, los TSAP con grado universitario de una disciplina afín y/o un posgrado en audiología mostrarían un perfil profesional más cercano al AG que los profesionales con solo titulación en TSAP.

\section{Métodos}

\section{Cuestionario}

Se elaboró un cuestionario en la plataforma de formularios de Google dirigido a TSAP que ejercen su labor profesional en España (Ver PDF adjunto "Sup. Material"), el cual se difundió en línea. Los participantes completaron el cuestionario de forma voluntaria y anónima, y no recibieron compensación por su participación. El estudio fue aprobado por el Comité de Ética de la Investigación de Universidad de Vanderbilt (EE. UU).

El cuestionario se dividió en tres secciones: preguntas demográficas (7), preguntas sobre desempeño profesional (12) y preguntas sobre formación en audiología (12). Las preguntas demográficas incluyeron la edad de los participantes, el sexo, la educación y la formación recibida, la comunidad autónoma donde ejercían como TSAP y el/los lugar/es actual/es de trabajo. Los lugares de trabajo se dividieron en centros auditivos (independientes y cadenas), ópticas $^{4}$ (independientes y cadenas), centros de atención temprana, centros de enseñanza (institutos, universidades o centros privados), fabricantes del sector, hospitales o clínicas de ORL privados, hospitales públicos y situación de desempleo.

Las preguntas sobre desempeño profesional se centraron en las poblaciones atendidas (pediátricas o adultas) y el tipo de servicio ofrecido (asesoramiento y adaptación de audífonos e implantes, y evaluación y tratamiento de trastornos vestibulares). Además, se incluyeron preguntas sobre la aplicación de buenas

\footnotetext{
${ }^{4}$ En España las ópticas tienen autorización para dispensar audífonos, por
} lo que muchos TSAP trabajan en este tipo de establecimientos sanitarios. prácticas clínicas basadas en la evidencia, concretamente la realización de mediciones en oído real a la hora de adaptar audífonos. Esta pregunta es característica de cuestionarios de desempeño profesional y se utiliza como medidor de buenas prácticas, ya que la realización de este protocolo clínico predice la satisfacción del paciente con la adaptación protésica (Aarts \& Caffee, 2005; Kirwood, 2010; Mueller \& Picou, 2010). Las respuestas de desempeño profesional se organizaron en una escala de Likert de uno a cuatro ( 1 = nunca, 4 = siempre).

Las preguntas de formación se centraron en las percepciones de los profesionales sobre su formación general y en áreas específicas de la audiología (asesoramiento y adaptación de audífonos, implantes osteointegrados, y asesoramiento y programación de implantes cocleares). Además, se les preguntó cómo percibirían la creación de un título oficial de grado universitario en audiología en España, si pensaban que la formación de audiología en España estaba al mismo nivel que otros países y si, en general, sentían estar formados para las tareas que desempeñaban en su práctica clínica diaria. Las respuestas a las preguntas de la sección de formación se organizaron en una escala de tipo Likert de uno a cuatro ( 1 = totalmente en desacuerdo, 4 = totalmente de acuerdo). Por último, cabe destacar que en el presente estudio analizamos una submuestra de las preguntas incluidas en el cuestionario que son pertinentes para responder a nuestras preguntas de investigación. Los resultados de las preguntas no incluidas en este estudio están disponibles bajo petición.

\section{Participantes}

Doscientos noventa y dos participantes cumplimentaron el cuestionario entre mayo y diciembre de 2018. De estos, 33 indicaron no estar en posesión del título de TSAP, por lo que sus respuestas fueron descartadas. De la muestra restante se descartaron otros cuatro cuestionarios porque los profesionales informaron ejercer actualmente fuera de España (tres en América y uno en el Reino Unido). Como resultado, se seleccionaron 255 cuestionarios para este estudio: 176 fueron completados por mujeres y 79 por hombres. El grupo de edad más representado fue el de 25-35 años con 102 participantes, seguido del de 35-45 años con 90 participantes, el de 45-55 años con 37 participantes, el de 18-25 años con 17 participantes y el de $>55$ años con 9 participantes. Las comunidades autónomas de procedencia están representadas en la 
tabla 1. Además, del total de 255 TSAP, 186 (el 72\%) refirieron no tener titulación adicional al ciclo superior (TSAP), 11 participantes (el 4\%) obtuvieron complementariamente al ciclo superior en TSAP un posgrado en audiología ${ }^{5}($ TSAP+P), 41 (el 16\%) un grado universitario de una disciplina afín a la audiología (TSAP+U) y 17 (el 7\%) un grado universitario de una disciplina afín y un posgrado en audiología (TSAP+UP).

Tabla 1: Distribución de los participantes por comunidad autónoma

\begin{tabular}{|l|l|}
\hline Comunidad autónoma & Porcentaje (\%) \\
\hline Comunidad de Madrid & 27 \\
\hline Comunidad Valenciana & 14 \\
\hline Andalucía & 13 \\
\hline Cataluña & 9 \\
\hline Castilla y León & 6 \\
\hline País Vasco & 6 \\
\hline Otras & 25 \\
\hline $\begin{array}{l}\text { Notas. La pregunta sobre comunidades autónomas fue } \\
\text { añadida al cuestionario después de su lanzamiento, por lo } \\
\text { que solo están representados 159 participantes. Cantabria, } \\
\text { Ceuta y Melilla no aparecieron representadas en el } \\
\text { cuestionario. }\end{array}$ \\
\hline
\end{tabular}

\section{Análisis}

Para analizar los lugares de trabajo se computaron el número total de respuestas para cada categoría de esta pregunta (cada participante pudo marcar más de un lugar de trabajo), así como el porcentaje de respuestas en cada categoría de lugar de trabajo sobre el total de respuestas. Para analizar si había diferencias entre los grupos divididos por nivel educativo (TSAP, TSAP+U, TSAP+P, TSAP+UP) en cuanto a sus lugares de trabajo más comunes, se llevó a cabo una prueba de la $\chi^{2}$ de heterogeneidad con simulación de Montecarlo con las categorías de lugar de trabajo como variable dependiente y los grupos por nivel educativo como variable independiente. Además, se realizaron análisis de la $\chi^{2}$ a posteriori para analizar qué grupos en concreto diferían entre sí en la distribución total de lugares de trabajo.

Para analizar si existían diferencias entre los grupos en cuanto a los servicios ofrecidos y la percepción sobre su formación, se llevaron a cabo análisis de varianza (ANOVA, por sus sigas en inglés) interindividuales de vía única para cada una de las preguntas de servicios y de formación. En todos los ANOVA, la Algunos títulos propios de postgrado en audiologĺa de universidades espa-
ñolas admitían en sus cursos a TSAP sin titulación universitaria durante los primeros años de implantación. variable dependiente fue la puntuación dada para cada pregunta (entre 1 y 4) y la variable independiente interindividual, el grupo por nivel educativo (TSAP, TSAP+U, TSAP+P, TSAP+UP). En todos los casos, cuando los resultados de los ANOVA globales (ómnibus) se mostraron significativos, se realizaron análisis a posteriori de comparaciones por pares para analizar diferencias entre los distintos grupos. En casos de violación de los supuestos de esfericidad, los valores de $p$ de los ANOVA se computaron con corrección de Huynh-Feldt. Por último, en todos los análisis a posteriori se utilizó la corrección de comparaciones múltiples de falso descubrimiento de Benjamini y Hochberg.

\section{Resultados}

\section{Lugares de trabajo}

En general, teniendo en cuenta la totalidad de la muestra, observamos que los centros de trabajo donde los participantes indicaron desarrollar su labor profesional con mayor frecuencia fueron los centros auditivos u ópticas (el 82\%); otros lugares de trabajo indicados fueron clínicas privadas de ORL u hospitales (privados y públicos; el 6\%), empresas o laboratorios del sector (el 5\%), centros de enseñanza (el 3\%) y centros de atención temprana (el $2 \%$ ). Tan solo un $2 \%$ del total de la muestra indicó estar desempleado (ver la tabla 2). Los grupos por nivel educativo de la muestra (TSAP, TSAP+U, TSAP+P y TSAP+UP) difirieron significativamente en cuanto a sus lugares de trabajo $\left(\chi^{2}=60,84 p \quad 0,01\right)$. Los análisis a posteriori indicaron que esta diferencia se encontraba entre el grupo TSAP+UP y el grupo TSAP $(p<0,01)$ y entre el grupo TSAP+UP y el grupo TSAP+U $(p<0,01)$. Por ejemplo, los TSAP+UP trabajaron más en centros de atención temprana (el 10,5\%) u hospitales (el 5\%) que el resto de los grupos (el 0\%; tabla 2).

\section{Servicios de audiología}

En general, teniendo en cuenta la totalidad de la muestra, observamos que una amplia mayoría de los participantes indicó realizar con frecuencia asesoramiento y adaptación de audífonos (el 92\%), seguido por asesoramiento y adaptación de implantes osteointegrados (el 23\%), implantes cocleares (el 14,5\%) y diagnóstico y tratamiento de problemas vestibulares (el 10\%; ver la figura 1). Además, la mayoría de los participantes indicó dar servicio a pacientes adultos (el 93\%), mientras que el número de participantes que indica- 
Tabla 2: Porcentaje de respuestas para cada centro de trabajo por nivel educativo

\begin{tabular}{|c|c|c|c|c|c|}
\hline & \multirow{2}{*}{$\begin{array}{l}\text { Muestra Completa } \\
\qquad(\mathrm{n}=255)\end{array}$} & \multicolumn{4}{|c|}{ Grupos por nivel de formación } \\
\hline & & $\begin{array}{l}\text { TSAP } \\
(n=186)\end{array}$ & $\begin{array}{l}\text { TSAP+U } \\
(n=41)\end{array}$ & $\begin{array}{l}\text { TSAP+P } \\
(n=11)\end{array}$ & $\begin{array}{l}\text { TSAP+UP } \\
(n=17)\end{array}$ \\
\hline $\begin{array}{l}\text { Óptica } \\
\text { (cadena, } 5 \text { centros o más) }\end{array}$ & $14,0 \%$ & $15,0 \%$ & $15,5 \%$ & $15,5 \%$ & $0,0 \%$ \\
\hline $\begin{array}{l}\text { Óptica independiente } \\
\text { ( } 5 \text { centros o menos) }\end{array}$ & $10,5 \%$ & $8,0 \%$ & $24,5 \%$ & $0,0 \%$ & $5,0 \%$ \\
\hline $\begin{array}{l}\text { Centro auditivo } \\
\text { (cadena, } 5 \text { centros o más) }\end{array}$ & $31,0 \%$ & $35,5 \%$ & $24,5 \%$ & $15,5 \%$ & $10,5 \%$ \\
\hline $\begin{array}{l}\text { Centro auditivo independiente } \\
\text { ( } 5 \text { centros o menos) }\end{array}$ & $27,0 \%$ & $24,0 \%$ & $22,5 \%$ & $46,0 \%$ & $53,0 \%$ \\
\hline Centro de atención temprana & $2,0 \%$ & $1,0 \%$ & $2,0 \%$ & $0,0 \%$ & $10,5 \%$ \\
\hline Hospital o clínica ORL privados & $5,0 \%$ & $5,5 \%$ & $2,0 \%$ & $7,5 \%$ & $5,0 \%$ \\
\hline Hospital público & $0,5 \%$ & $0,0 \%$ & $0,0 \%$ & $0,0 \%$ & $5,0 \%$ \\
\hline Centro de enseñanza (audiología) & $3,0 \%$ & $2,5 \%$ & $4,5 \%$ & $0,0 \%$ & $10,5 \%$ \\
\hline Fabricante/laboratorio del sector & $5,0 \%$ & $6,0 \%$ & $2,5 \%$ & $15,5 \%$ & $0,0 \%$ \\
\hline Desempleado & $2,0 \%$ & $2,5 \%$ & $2,0 \%$ & $0,0 \%$ & $0,0 \%$ \\
\hline \multicolumn{6}{|c|}{$\begin{array}{l}\text { Notas. TSAP: técnico superior en audiología protésica, TSAP+U: técnico superior en audiología protésica con grado universitario en } \\
\text { una disciplina afín a la audiología, TSAP+P: técnico superior en audiología protésica con posgrado en audiología, TSAP+UP: técnico } \\
\text { superior en audiología protésica con grado universitario en una disciplina afín y posgrado en audiología. Los porcentajes pueden } \\
\text { sumar más del } 100 \% \text {, ya que los participantes podían indicar más de un centro de trabajo. }\end{array}$} \\
\hline
\end{tabular}

ron atender con frecuencia a pacientes menores de 18 y menores de 5 años fue inferior (el 34\% y el 20\%, respectivamente; ver la figura 2 ). Un $65 \%$ de la muestra indicó llevar a cabo medidas en oído real a la hora de adaptar audífonos (buenas prácticas). Por último, un $22 \%$ de la muestra indicó asesorar con frecuencia a sus pacientes sobre los distintos métodos de comunicación (lenguaje oral, lengua de signos, etc.). Los ANOVA realizados para comparar los tipos de servicios ofrecidos entre los grupos por nivel educativo revelaron diferencias significativas en algunas de las preguntas. Concretamente, los grupos difirieron significativamente en la frecuencia con la que ofrecían asesoramiento y programación sobre implantes cocleares $\left(F_{3,251}=3,5, p<0,05, \eta_{G}{ }_{G}=0,04\right)$ y en la frecuencia con la que aportaban información sobre opciones de comunicación $\left(F_{3,251}=6,8, p<0,001, \eta_{G}^{2}=0,08\right)$ a sus pacientes. Los análisis a posteriori revelaron que los TSAP+UP proveían significativamente menos servicios relacionados con el asesoramiento y programación de implantes cocleares que los TSAP $(p<0,05)$ y los TSAP+U $(p<0,05)$. Además, los participantes con TSAP+UP proporcionaban información con mayor frecuencia a sus pacientes sobre opciones de comunicación que los TSAP $(p<0,001)$ y que los TSAP $+U(p<0,05)$. No se encontraron diferencias entre ninguno de los grupos en el resto de las preguntas sobre servicios ofrecidos (audífonos, implantes osteointegrados o trastornos del equilibrio; ver la figura 1 ).
Los ANOVA realizados para comparar los tipos de servicios ofrecidos por edades entre los grupos por nivel educativo revelaron diferencias significativas en cuanto al número de menores de cinco años atendidos $\left(F_{3,251}=4,4, p<0,01, \eta_{G}^{2}=0,05\right)$. Los análisis a posteriori revelaron que los TSAP+UP atendían con una frecuencia significativamente más alta a menores de cinco años que los TSAP $(p<0,01)$ y los $\mathrm{TSAP}+\mathrm{U}(p<0,05)$. No se encontraron diferencias entre ninguno de los grupos en el resto de las preguntas sobre servicios por edades (adultos y menores de 18 años; ver la figura 2).

\section{Percepción sobre formación}

En general, teniendo en cuenta la totalidad de la muestra observamos que un alto porcentaje de participantes (el 87\%) indicaron tener la suficiente formación para ejercer sus labores audiológicas diarias. Sin embargo, tan solo un $26 \%$ indicó que la formación en audiología en España estaba al nivel de otros países del entorno. En cuanto a la educación recibida, casi todos los participantes (el 94,5\%) indicaron percibir tener una formación adecuada para evaluar, asesorar y adaptar a los pacientes con audífonos. Por el contrario, un $36 \%$ y un $21 \%$ de los participantes señaló haber recibido la formación adecuada para evaluar, asesorar, adaptar y programar implantes osteointegrados e implantes cocleares, respectivamente. Además, un elevado porcentaje de la muestra 


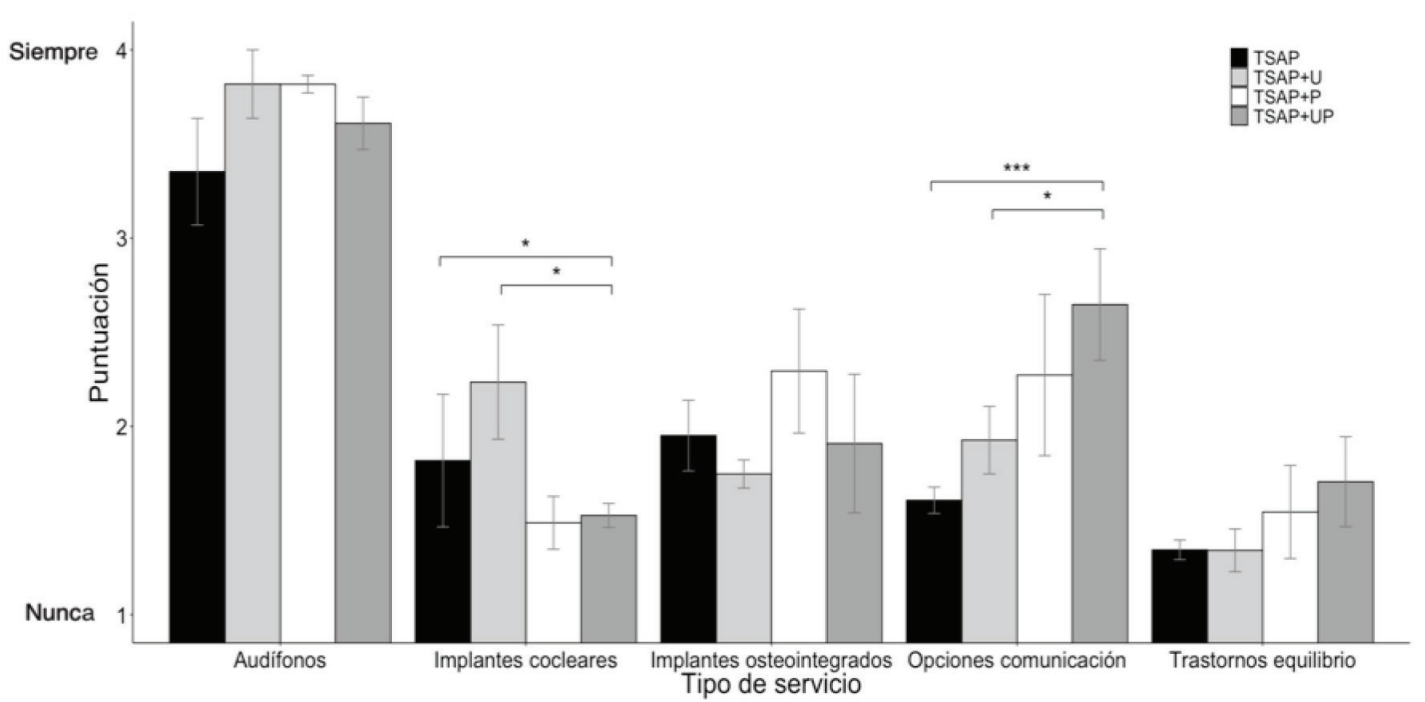

Figura 1. Puntuación media provista por los grupos de técnico superior en audiologla protésica de la muestra en las preguntas del cuestionario relacionadas con los servicios audiológicos. TSAP: técnico superior en audiologla protésica, TSAP+U: técnico superior en audiologĺa protésica con grado universitario en una disciplina afín a la audiologĺa, TSAP+P: técnico superior en audiologĺa protésica con posgrado en audiologĺa, TSAP+UP: técnico superior en audiologĺa protésica con grado universitario en una disciplina afín y posgrado en audiologĺa. ${ }^{*} p<0,05 ;{ }^{* * *} p<0,001$.

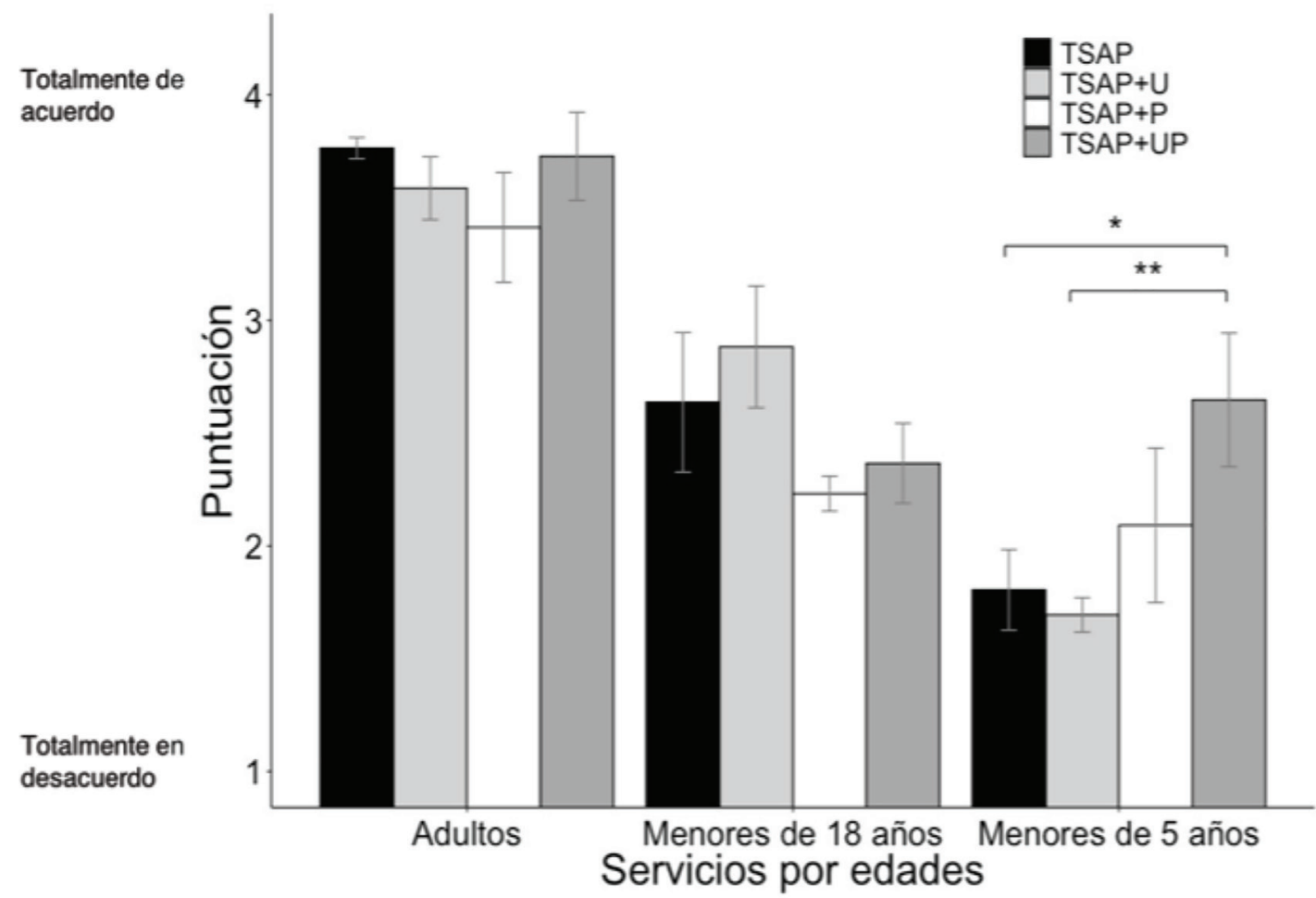

Figura 2. Puntuación media provista por los grupos de técnico superior en audiologla protésica de la muestra en las preguntas del cuestionario relacionadas con los servicios ofrecidos por edades. TSAP: técnico superior en audiologla protésica, TSAP+U: técnico superior en audiologla protésica con grado universitario en una disciplina afín a la audiologĺa, TSAP+P: técnico superior en audiologla protésica con posgrado en audiologĺa, TSAP+UP: técnico superior en audiologla protésica con grado universitario en una disciplina afín y posgrado en audiologĺa. ${ }^{*} p<0,05 ;{ }^{* *} p<0,01$.

reportó tener la suficiente formación para poner en práctica información científica en su trabajo diario (el $65 \%$ ) y buscar oportunidades de formación continua (el 89\%). Además, la mayoría de los participantes (el 90\%) indicó que en España era necesario un grado en audiología y que lo intentarían cursar si llegase a existir (el 94,5\%). Por último, un $23 \%$ de los participantes señaló que la existencia de un grado en audiología supondría una amenaza para su puesto de trabajo. 
Los ANOVA realizados para comparar la percepción sobre la formación entre los grupos por nivel educativo reveló diferencias significativas en cuanto a la formación en programación y asesoramiento sobre implantes cocleares $\left(F_{3,251}=4,5, p<0,01, \eta^{2}{ }_{G}=0,05\right.$; ver la figura 3). Los análisis a posteriori revelaron que los TSAP+UP percibían tener una mejor formación en implantes cocleares que los $\operatorname{TSAP}(p 0,01)$ y los TSAP+U $(p<0,01)$. No se encontraron diferencias entre ninguno de los grupos en el resto de las preguntas sobre formación (audífonos e implantes osteointegrados). Por lo tanto, no existieron diferencias significativas entre los grupos a la hora de aplicar buenas prácticas clínicas, buscar formación continua o en su percepción sobre la necesidad de crear o no un grado de audiología en España, entre otros.

\section{Discusión}

El propósito de este estudio fue conocer si los distintos perfiles profesionales por nivel educativo de los técnicos superiores en audiología protésica (TSAP) españoles diferían en los lugares de trabajo, los servicios ofrecidos y la percepción sobre su formación, y examinar si alguno de estos perfiles profesionales realizaba labores propias del audiólogo general (AG).
Comparamos cuatro grupos de profesionales: TSAP con solo ciclo superior (TSAP), TSAP con grado universitario de una disciplina afín a la audiología (TSAP+U), TSAP con posgrado universitario (TSAP+P) y TSAP con grado universitario en una disciplina afín y posgrado en audiología (TSAP+UP). Los resultados indicaron que, con independencia de su nivel educativo, la mayoría de los TSAP españoles (1) no tienen formación universitaria en forma de grado universitario de una disciplina afín o posgrado en audiología (el 71\%), (2) desempeñan su labor profesional en centros auditivos y ópticas, (3) limitan su labor profesional a la adaptación de audífonos a personas adultas, para lo que percibieron estar bien formados, y (4) no muestran un perfil profesional de AG, ya que de acuerdo con la Federación Europea de Sociedades Audiológicas (EFAS, 2001), las competencias del AG se extienden al tratamiento de la pérdida auditiva con implantes osteointegrados e implantes cocleares, así como el diagnóstico y tratamiento de acúfenos y trastornos vestibulares.

\section{Diferencias entre los grupos por nivel educativo: lugares de trabajo}

Con independencia del nivel educativo, la mayoría de Ios TSAP desarrollaron su labor profesional en centros auditivos u ópticas. El porcentaje de TSAP que ejercen en otros lugares de trabajo fue menor al $20 \%$. Sin

Figura 3. Puntuación media provista por los grupos de técnico superior en audiologla protésica de la muestra en las preguntas del cuestionario relacionadas con la percepción de la formación por áreas audiológicas. TSAP: técnico superior en audiologla protésica, TSAP+U: técnico superior en audiologla protésica con grado universitario en una disciplina afín a la audiologĺa, TSAP+P: técnico superior en audiologĺa protésica con posgrado en audiologĺa, TSAP+UP: técnico superior en audiologĺa protésica con grado universitario en una disciplina afín y posgrado en audiologla. ** $p<0,01$.

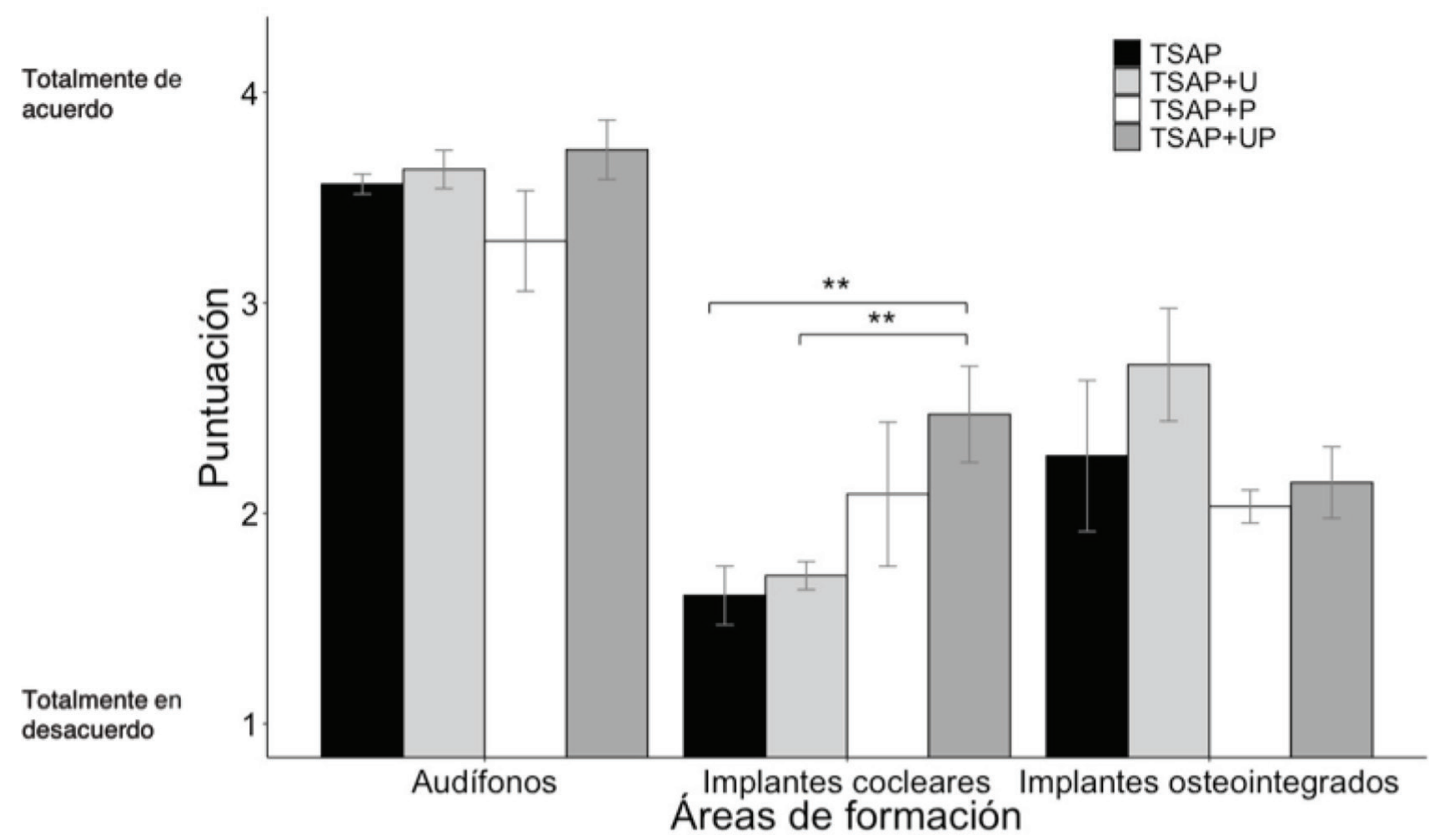


embargo, y parcialmente de acuerdo con nuestra hipótesis, observamos que los TSAP+UP trabajaban en mayor proporción que los TSAP y los TSAP+U en centros auditivos independientes y hospitales públicos. Los TSAP y los TSAP+U trabajaban en mayor proporción en centros auditivos de cadenas comerciales y ópticas que el resto de los grupos. Esto es de interés porque, pese a centrarse también en la adaptación de audífonos, los centros auditivos independientes tienden a atender a pacientes con necesidades más específicas (p. ej., población pediátrica) que las ópticas y centros auditivos de cadenas comerciales, los cuales atienden principalmente a población mayor con pérdida auditiva. Es posible que los centros auditivos independientes y hospitales busquen activamente contratar a TSAP con mayor nivel educativo, los cuales pueden proveer servicios más especializados a sus pacientes. Pese a que los TSAP+UP están presentes en hospitales, el porcentaje es bajo (el 5\%). Sería interesante investigar qué profesionales están llevando a cabo las labores del AG propuesto por EFAS en hospitales, clínicas de ORL o centros de atención temprana. Los estudios futuros podrían estudiar la formación y competencias de otros profesionales de la salud auditiva, así como la satisfacción de los pacientes con los servicios recibidos para valorar la posibilidad de realizar mejoras en la educación en audiología en España.

\section{Diferencias entre los grupos por nivel educativo: servicios of recidos y formación}

Solo observamos diferencias entre los grupos en dos preguntas relacionadas con servicios y formación. La primera diferencia la detectamos en la atención a pacientes menores de cinco años, los cuales son atendidos con más frecuencia por los TSAP+UP que por los TSAP y los TSAP+U. Esto puede estar relacionado con el hecho de que los TSAP con mayor nivel educativo estén más presentes en centros auditivos especializados, centros de atención temprana y hospitales públicos que los que tienen menor nivel educativo. La segunda diferencia la encontramos en la programación de implantes cocleares, donde los TSAP+UP indicaron sentirse más formados que los TSAP y los TSAP+U. Pese a ello, los TSAP+UP ofrecieron servicios en esta área con menor frecuencia que los otros dos grupos. Esto podría explicarse por el hecho de que algunos centros auditivos de grandes cadenas (donde existe una mayor prevalencia de TSAP sin posgrado en audiología) proveen servicios de programación de implantes cocleares. Por lo tanto, es posible que algunos profesionales (TSAP y $\mathrm{TSAP}+\mathrm{U}$ ) tengan que asesorar y programar implantes cocleares pese a que no perciben tener la formación adecuada para ello. De cualquier modo e independientemente de su nivel educativo, la frecuencia con la que los profesionales ofrecieron servicios de programación de implantes fue baja en todos los grupos. Por lo tanto, es posible que otros profesionales de la salud auditiva (ORL, ingenieros de casas de implantes, etc.) estén llevando a cabo esta labor propia del AG. Futuros estudios deberán encargarse de definir exactamente quién realiza las programaciones de implantes cocleares, la formación que reciben y la calidad del servicio. En general, la amplia mayoría de los TSAP percibieron estar formados principalmente para adaptar audífonos a adultos e indicaron ofrecer con más frecuencia este servicio que cualquier otro.

Por último e independientemente del nivel educativo, los profesionales indicaron que el nivel de formación en audiología en España no estaba al nivel de otros países del entorno y que veían necesaria la creación de un grado universitario en audiología. Es probable que estas respuestas estén motivadas por la falta de formación que indicaron los profesionales en ciertas áreas de la audiología. Sin embargo, la mayoría de los participantes indicaron sentirse suficientemente formados para llevar a cabo sus tareas clínicas diarias, las cuales se centran en la adaptación de audífonos a pacientes adultos. Además, el porcentaje de participantes que indicó llevar a cabo de forma regular buenas prácticas utilizando medidas en oído real es similar al reportado en países como Reino Unido o Estado Unidos ( 65\%; Aarts \& Caffee, 2005; Kirwood, 2010; Mueller \& Picou, 2010).

Por lo tanto, en base a los datos de esta encuesta, el sistema de formación en audiología en España no está en condiciones de producir un perfil profesional que se asemeje al modelo de audiólogo general. Los estudios futuros podrían incluir a profesionales de disciplinas afines a la audiología (ingenieros, ORL, logopedas y otro personal de la salud) para ofrecer una visión global sobre la situación de la audiología en España. Es necesario que las asociaciones nacionales de audiología lleven a cabo estudios que incluyan a todos los profesionales, su formación y los servicios que ofrecen, así como la satisfacción de los pacientes. Dependiendo de los resultados obtenidos, podría considerase, y quizás fomentarse, la creación de un grado universitario en audiología en España complementario al actual ciclo superior en TSAP, a través 
del cual pudieran formarse AG siguiendo el modelo propuesto por EFAS. De esta manera, podría surgir y reconocerse la figura del AG, lo cual abriría ámbitos de actuación profesional que actualmente parecen inaccesibles para los TSAP. Con ello se podrían mejorar potencialmente los servicios de salud auditiva.

\section{Limitaciones}

Los resultados de este estudio se basaron en la percepción de los profesionales en TSAP usando un cuestionario digital y no se obtuvieron observaciones directas sobre su desempeño profesional. Pese a ser una práctica muy común analizar el desempeño profesional a través cuestionarios (Aarts \& Caffee, 2005; Kirwood, 2010; Mueller \& Picou, 2010), es importante recordar que los cuestionarios tienen sus limitaciones. La principal es el sesgo de aceptación social que provoca que los encuestados tiendan a contestar de una manera socialmente aceptable. Algunas de las estrategias que previenen el sesgo de aceptación social es informar a los encuestados de que los resultados son anónimos, usar un cuestionario en lugar de una entrevista o usar respuestas de múltiple elección en lugar de respuestas abiertas: todas estas estrategias se incluyeron en el cuestionario usado en este estudio. Además, este estudio está limitado por las diferencias de tamaño de las muestras entre los grupos, lo cual podría afectar la potencia estadística para detectar diferencias significativas entre los grupos. Por lo tanto, la interpretación de los resultados tiene que tomarse con cautela hasta que los futuros estudios repliquen estos hallazgos.

\section{Conclusión}

Los datos de este estudio indican que, independientemente de si han completado formación universitaria en forma de grado universitario de una disciplina afín o posgrado en audiología, la mayoría de los TSAP en España desarrollan su labor profesional adaptando audífonos a pacientes adultos en centros auditivos. Los TSAP con posgrado universitario en audiología y grado universitario de una disciplina afín reciben una formación más especializada, lo cual les permite trabajar con mayor frecuencia en centros auditivos independientes y en algunos casos en centros de atención temprana y hospitales. Sin embargo, esta formación no les permite llevar a cabo, de forma generalizada, labores propias del AG, como pueden ser la programación de implantes cocleares, implantes osteointegrados, trastornos del equilibrio o atención a pacientes pediátricos. Por lo tanto, la formación en TSAP (con o sin posgrado en audiología) no genera profesionales capaces de desarrollar las labores propias del AG. La situación de la audiología en España necesita un análisis más detallado para valorar la calidad de los servicios ofrecidos a los pacientes con problemas auditivos, así como posibles cambios en la formación de los profesionales para adecuarse a las recomendaciones de EFAS.

\section{Referencias}

Aarts, N. L., \& Caffee, C. S. (2005). Manufacturer predicted and measured REAR values in adult hearing aid fitting: Accuracy and clinical usefulness. International Journal of Audiology, 44(5), 293-301. https://doi. org/10.1080/14992020500057830

American-Speech-Language-Hearing Association (2020, Mayo 8). Hearing and Balance. https://web.archive.org/web/20200508060253/https:// www.asha.org/public/hearing/

European Federation of Audiology Societies (EFAS). (2001) Audiology in Europe. Recuperado de https://web.archive.org/web/20191224091707/ http://www.efas.ws/noticeboard/general_audiologist.htm. Último acceso el 20 de abril de 2020.

Goulios, H. (2010). Comparative audiology and the development of a seeding model for affordable and sustainable audiology education (Tesis Doctoral, University of Western Australia, Australia). Recuperado de https://research-repository.uwa.edu.au/en/publications/comparative-audiology-and-the-development-of-a-seeding-model-for-

Goulios, H., \& Patuzzi, R. B. (2008). Audiology education and practice from an international perspective. International Journal of Audiology, 47(10), 647-664. https://doi.org/10.1080/14992020802203322

Kirkwood, D. H. (2010). Survey probes dispensers' views on key issues raised by consumer reports. The Hearing Journal, 63(5), 17-18. https:// doi.org/10.1097/01.HJ.0000373446.75826.e1

Mueller, H. G., \& Picou, E. M. (2010). Survey examines popularity of realear probe-microphone measures. The Hearing Journal, 63(5), 27-28. https://doi.org/10.1097/01.HJ.0000373447.52956.25

Orden SC0/1262/2007, de 13 de abril, por la que se aprueba y publica el programa formativo de la especialidad de Otorrinolaringología. Boletín Oficial del Estado núm. 110, de 8 de mayo de 2007, 19880 a 19889. https://www.boe.es/eli/es/o/2007/04/13/sco1262

Real Decreto 1685/2007, de 14 de diciembre, por el que se establece el título de Técnico Superior en Audiología Protésica y se fijan sus enseñanzas mínimas. Boletín Oficial del Estado núm. 13, de 15 de enero de 2008, 2615 a 2638. https://www.boe.es/eli/es/rd/2007/12/14/1685 


\section{Material suplementario}

\section{https://journal.auditio.com/auditio/libraryFiles/downloadPublic/16}

Conflictos de interés

Los autores declaran no tener ningún conflicto de interés.

Este estudio de investigación se llevó a cabo en ausencia

de relaciones comerciales o financieras que pudieran

interpretarse como posible conflicto de interés.

\section{Fondos}

Esta investigación no ha recibido fondos externos.

Declaración de disponibilidad de datos

Los datos no están disponibles públicamente.

Contribuciones de los autores:

Carlos Benítez Barrera y Beatriz de Diego Lázaro han contribuido por igual en la conceptualización, recogida de datos, borrador, revisión, edición del manuscrito, análisis de datos, metodología y visualización.

Agradecimientos

Agradecemos a Javier Santos la conceptualización del estudio, así como su participación activa en el desarrollo de la encuesta y la recogida de datos. Además, agradecemos a Anne Marie Tharpe su disposición a constar como tutora en el proceso de autorización del estudio por parte del Comité de Ética de la Investigación de la Universidad de Vanderbilt. 